\title{
Una Mirada a la Calidad de Vida y Salud de las Educadoras de Párvulos
}

\author{
A LOOK TO THE LIFE AND HEALTH QUALITY OF KINDERGARTEN TEACHERS
}

Patricia Arteaga González ${ }^{1}$, Alicia Hermosilla-Ávila², Carmen Mena Bastías ${ }^{3}$, Sonia Contreras Contreras²

1. Facultad de Educación y Humanidades, Departamento de Ciencias de la Educación. Universidad del Bio-Bío, Chillán, Chile.

2. Facultad de Ciencias de la Salud y los Alimentos, Departamento de Enfermería, Universidad del Bío-Bío, Chillán, Chile.

3. Facultad de Educación y Humanidades, Departamento de Ciencias de la Educación. Universidad del Bío-Bío, Chillán, Chile.

\begin{abstract}
RESUMEN
Introducción: La medición de la calidad de vida desde la perspectiva de la salud y su relación en el ámbito laboral de las Educadoras de Párvulos como agentes fundamentales en el desarrollo cognitivo y socioemocional en la primera infancia es de relevancia, pues existen aspectos claves para conseguir una buena calidad pedagógica en concordancia con los anhelos de las nuevas políticas en educación implementadas en Chile. Objetivo: determinar con qué factores se asocian la calidad de vida relacionada con la salud de las Educadoras de Párvulos, desde los elementos sociodemográficos y laborales concernientes en sus actividades profesionales. Método: Diseño metodológico descriptivo, correlacional, de corte transversal en 156 Educadoras de Párvulos de una comuna de Chillán, Chile. Resultados: las Educadoras de Párvulos, que se desempeñan casi en su totalidad en el aula, refieren un rol físico, función física y rol emocional óptimo, con una regular vitalidad y función social, una buena salud mental y mal dolor corporal, con relaciones altamente significativas en la función fisica con el ausentismo y dependencia laboral, y significativa con el número de niños a cargo y años laborales. También altamente relacionados en forma significativa el dolor corporal y los días de ausentismo, significativamente la vitalidad y el número de niños a cargo, y el rol emocional con los días de ausentismo laboral, entre otros. Discusión: Estos antecedentes podrían ser determinantes en la orientación de las prácticas educativas y ponen en manifiesto que las nuevas disposiciones y políticas gubernamentales no aseguran condiciones que propicien una buena calidad de vida docente.
\end{abstract}

(González P, Hermosilla-Ávila A, BastíasC, Contreras S, 2018. Una Mirada a la Calidad de Vida y Salud de las Educadoras de Párvulos. Cienc Trab. Ene-Abr; 20 [61]: 42-47).

Palabras claves: CALIDAD DE VIDA, EDUCACIÓN DE PÁRVULOS, SALUD.

\section{ABSTRACT}

Introduction: the measurement of life quality from a health perspective and its relationship on the labor field of Kindergarten Teachers as main agents on the cognitive and socio emotional development in early childhood, is relevant, since there are key aspects in order to get a good pedagogical quality according to the desires of the new educational politics implemented in Chile. Objective: to determinate which factors are associated to the quality of life related to the health of Kindergarten Teachers, from sociodemographic and labor elements concerning their professional activities. Method: descriptive methodological design, correlational, cross sectional in 156 Kindergarten Teachers from a commune in Chillán, Chile. Results: the Kindergarten Teachers, who mostly perform in the classroom, relate a physical role, physical function and an optimal emotional role, with a regular vitality and social function, a good mental health and body ache, with highly meaningful relations in the physical function with absenteeism and labor dependency. Also, body ache and days of absenteeism are highly related in a meaningful way, as well as vitality and the amount of kids they are in charge of, and the emotional role with the days of labor absenteeism, among others. Discussion: these antecedents may be determinant on the guidance of the teaching practices and show that the new regulations and governmental politics do not ensure conditions that promote good quality in docent life.

Key words: WORKING CONDITIONS, PRESCHOOL EDUCATION, QUALITY OF LIFE.
Correspondencia / Correspondence

Patricia Arteaga González

Facultad de Educación, Depto. Ciencias de la Educación.

Universidad del Bío-Bío

Avda. Brasil \#1180, Chillán, Chile.

e-mail: parteaga@ubiobio.cl

Recibido: 25 de Noviembre de 2017 / Aceptado: 08 de Marzo de 2018

\section{INTRODUCCIÓN}

La medición de la calidad de vida en cualquier contexto social es un reto, debido a que debe considerarse como un constructo multidimensional, de carácter dinámico, complejo, subjetivo y variable en el tiempo. ${ }^{1-3}$ La organización mundial de la salud en 1994 establece que la calidad de vida relacionada con la salud es "la percepción del individuo de su posición en la vida en el contexto de la cultura y sistema de valores en la que vive y en relación a sus objetivos, expectativas, estándares y preocupaciones". 4 Por ende, se refiere igualmente al bienestar físico, psíquico, espiritual y social de las personas, de su historia personal y la capacidad de las mismas para desenvolverse y desarrollar las tareas típicas de la vida cotidiana..$^{5,6}$ 
La calidad de vida relacionada con la salud en su asociación con el nivel de bienestar que tiene la persona en diversos dominios de su vida, considera el impacto que finalmente esos dominios pudieran alterar su estado de salud. Alteraciones que pueden ser resultado de una combinación de factores, tales como la utilización y aprovechamiento de los potenciales personales, como el intelecto, la emocionalidad y la creatividad, y de las circunstancias externas como a la estructura socioeconómica, sociopsicológica, cultural y política, que interactúan con el individuo. Como así también de aspectos subjetivos como la satisfacción a nivel personal y profesional. ${ }^{7-9}$

En el desarrollo profesional y como elemento central en la estructuración social está el trabajo, esencial para las personas y las sociedades, como esfera vital para los procesos de socialización, realización personal y subjetividad del individuo ${ }^{10}$, por ende, si existen ciertas circunstancias o factores de riesgo en el ambiente laboral, inevitablemente repercutirán en la salud de la persona, tales factores incluso a nivel estructural, de las exigencias diarias, de las posibilidades de ocio, de la posibilidad de desarrollo personal y social dentro del marco del trabajo. ${ }^{11}$

Pensando que el capital humano es el elemento más valioso de una organización para alcanzar sus objetivos, crece el interés por las distintas agrupaciones de trabajadores, y la observación de todos aquellos elementos relacionados con la productividad de los mismos, como su desmotivación, insatisfacción y desempeño deficiente, además de otros aspectos propios de la institución, como el sistema de trabajo, las políticas, los métodos de dirección y las estrategias organizacionales. ${ }^{12,13}$

Pues bien, así como la Educación Parvularia ha pasado a ser una prioridad, que busca impulsar una formación de calidad en los niños en sus primeros años, y promover la formación y actualización permanente de sus docentes responsables ${ }^{14}$, también es prioritario conocer más sobre las consecuencias de las condiciones laborales de las Educadoras de Párvulos, que no están exentas de elementos de preocupación que pudiesen afectar su calidad de vida relacionada con la salud. Como docentes en general, es conocido su alto nivel de malestar psicológico y alta prevalencia de trastornos de salud física y mental ${ }^{15}$, condición particularmente importante si pensamos que el estado de salud influye significativamente en el desempeño cotidiano, cuando tienes la responsabilidad de educar niños y niñas, ejerciendo una influencia determinante en la vida de estos. ${ }^{16}$

Conocer la calidad de vida en el trabajo recoge más importancia al representar el conjunto de acciones y factores que repercuten en la salud ocupacional, y en toda la multidimensionalidad del profesional, más aun en las Educadoras de Párvulos, que presentan complejas condiciones laborales en nuestro país, pues constituyen el grupo de profesionales con menores sueldos, altos niveles de estrés, escasos tiempos de distracción durante el día y sobrecarga que se mantiene durante toda la jornada laboral. ${ }^{1,17-19} \mathrm{Si}$ bien las actuales políticas dirigidas hacia la Educación Parvularia contemplan mejorar la atención hacia los niños, mejorar los espacios, infraestructura, seguridad y cobertura de los centros, a través de la recientemente creada Subsecretaria de Educación Parvularia como órgano de colaboración directa al Ministerio de Educación, aún falta direccionar los esfuerzos hacia sugerir estándares o referentes de condiciones laborales decentes para el personal profesional y técnico del área. ${ }^{20,21}$

Por todo ello, el principal objetivo del estudio es determinar con qué factores se asocian la calidad de vida relacionada con la salud de las Educadoras de Párvulos, desde los elementos sociodemográficos y laborales concernientes en sus actividades profesionales, para poder establecer diversos análisis respecto a las condiciones y la diversidad de las actividades desempeñadas por las Educadoras, aportando así a la discusión respecto al establecimiento de políticas que permitan mejorar las condiciones laborales y fortalecer la carrera docente.

\section{MATERIALES Y MÉTODOS}

Investigación del paradigma cuantitativo, con diseño metodológico descriptivo, correlacional, de corte transversal. Con el propósito de medir la calidad de vida relacionada con la salud de las Educadoras de párvulos y analizar sus implicancias como factor relevante para la salud laboral y el futuro impacto en su desempeño y rol como educador en la primera infancia. El universo en estudio corresponde a 156 Educadoras de Párvulos pertenecientes a establecimientos municipalizados y particulares subvencionados de la comuna de Chillán en Chile.

En relación a la caracterización general de la muestra, en su totalidad fueron mujeres, género predominante en esta profesión, de diversas edades, todas dependientes de establecimientos regulados directamente por el ministerio de educación con entidades públicas y particulares subvencionadas. La cantidad de participantes corresponde aproximadamente al 85\% de las Educadoras de Párvulos de la comuna de Chillán.

Los participantes respondieron un cuestionario en forma voluntaria otorgando consentimiento informado. Los datos recogidos mediante el cuestionario SF-26 previamente validado para medir calidad de vida relacionada con la salud a nivel mundial y latinoamericano, que consta de 36 items que exploran 8 dimensiones del estado de salud: función física, función social, problemas físicos, problemas emocionales, salud mental, vitalidad, dolor y percepción de la salud general, el cual fue autoadministrado y respondido en forma anónima. ${ }^{22,23}$

Tabla 1.

Características Cuestionario SF-36.

\begin{tabular}{|lcc|}
\hline Dimensión & No ítems & Puntaje \\
\hline Función física & 10 & $0-100$ \\
\hline Rol físico & 4 & $0-100$ \\
\hline Dolor corporal & 2 & $0-100$ \\
\hline Vitalidad & 5 & $0-100$ \\
\hline Salud general & 4 & $0-100$ \\
\hline Función social & 2 & $0-100$ \\
\hline Rol emocional & 3 & $0-100$ \\
\hline Salud mental & 5 & $0-100$ \\
\hline
\end{tabular}

Fuente: elaboración por autores.

Las puntuaciones que entrega el cuestionario en relación a cada una de las ocho dimensiones establecidas por el instrumento, oscilan entre los valores 0 y 100 . Siendo 100 un resultado que indica una salud óptima y 0 reflejaría un estado de salud muy malo, específicamente 0-20 como muy mala. 21-40 mala, 41-60 regular, 61-80 buena, 81-100 óptima. ${ }^{24}$

El instrumento a nivel nacional ha demostrado una buena confiabilidad en estudios, presentando un alfa de Cronbach en promedio de sus subescalas de 0,79 , sin un cálculo general debido a que el instrumento no genera un índice global, así como también una buena validez, siendo usado ampliamente en Sudamérica por diversos investigadores. ${ }^{25-27}$ 
Para el análisis estadístico de los datos, se utilizó el programa SPSS versión 21, determinando distribución de frecuencias, medidas descriptivas de tendencia central, y para determinar la asociación entre variables cualitativas el estadístico de chi-cuadrado, considerando una relación significativas con valores $\mathrm{p}$ menores a 0,05 y altamente significativas con valores p menores a 0,01.

En el análisis de fiabilidad de las dimensiones del instrumento de calidad de vida relacionada con la salud, para determinar el grado en que las respuestas a las preguntas se relacionan entre sí, para medir lo mismo y contribuyen a la medición global se usó alpha de Cronbach, obteniendo como fiabilidad del instrumento para la presente investigación en promedio de sus subescalas de 0,7 , valor relevante, considerando que sobre 0,7 se considera aceptable y respalda la consistencia interna de los ítems del cuestionario.

\section{RESULTADOS}

\section{Análisis descriptivo de la muestra}

Los 156 participantes en el estudio son de sexo femenino en su totalidad, y la edad de las educadoras en un 42,9\% oscila entre los 23 y 35 años, un 30,1\% entre los 36 y 45 años, un 23,7\% entre los 46 y 60 años, y solo un 3,2\% tiene más de 60 años.

En relación a los antecedentes laborales, el 71,8\% de las docentes encuestadas labora en los niveles de transición, es decir, trabajan con niños cuyas edades fluctúan entre los 4 y 6 años de edad, un 16,7\% en los niveles medios (con niños/as entre los 2 y 4 años) y un 11,5\% en los niveles de sala cuna (con niños/as entre 0 y 2 años).

Respecto a la dependencia laboral, que corresponde al organismo con el que las profesionales sostienen relación contractual, y que representa distintas condiciones laborales en cuanto a tipos de contrato, remuneraciones, jornadas de trabajo, entre otras condiciones, un 44,9\% pertenece al sistema particular subvencionado, un $23,7 \%$ corresponde al sistema de vía transferencia de fondos y un $31,4 \%$ al sistema municipalizado.

En relación a los años laborales activos, referido a la cantidad de tiempo que cada educadora lleva ejerciendo su rol frente a niños/ as, se aprecia que un 45,5\% tiene entre 1 y 10 años, un 31,4\% entre 11 y 20 años y un 23,1\% con más de 21 años. Respecto a la cantidad de niños/as que tienen a cargo las educadoras, un 58,3\% tienen entre 21 y 40 estudiantes, un 38,5\% tiene entre 7 y 20 estudiantes y un 3,2\% tiene sobre 40 niños/as.

En lo referente a la categoría de días de ausencia laboral por motivos de enfermedad, tales como dificultades osteomusculares, cardiológicas, respiratorias, metabólicas, psiquiátricas, entre otras, un $48,1 \%$ tiene entre 0 y 5 días de ausentismo, el 28,8\% entre 6 y 25 días, el 12,8\% entre 26 y 50 días y un 10,3\% con más de 50 días de ausencia, días que son una muestra tangible de cómo la salud se interrelaciona con su desempeño profesional.

\section{Análisis descriptivo del cuestionario}

Del análisis de las dimensiones del cuestionario SF-36 de calidad de vida relacionado con la salud, en promedio, se extrae que la función física, referida a las actividades diarias más comunes como bañarse, vestirse, caminar, agacharse, etc. o las más vigorosas como subir escaleras o correr es percibida como óptima (93 puntos), no hay casos con muy mala o mala función física. El $87,8 \%(n=137)$ presenta óptima función física y solo un 9,6\% $(\mathrm{n}=15)$ buena, situación que evidencia que la salud de la docente preescolar no limita la realización de sus actividades de la vida diaria.
De igual forma, el rol físico, relacionado con las dificultades percibidas respecto a cuánto afecta en el trabajo o en las actividades de la vida diaria el estado de salud general, también se percibe como óptimo (94 puntos); no hay casos muy malos ni malos, el 84\% ( $\mathrm{n}=$ 131) está óptimo y el 12,2\% $(n=19)$ está bueno, situación que podría implicar que el estado de salud de las profesionales no interfiere con su labor profesional.

El dolor corporal, referido a cómo este puede afectar o limitar actividades cotidianas, ya sea a nivel personal o laboral, es percibido como malo (40 puntos), el 27,6\% ( $\mathrm{n}=43$ ) tiene muy malo, el 30,1\% ( $\mathrm{n}=$ 47) malo, el $23,7 \%(n=37)$ regular y el $7,7 \%(n=12)$ óptimo, situación que podría implicar que existen aspectos relacionados con el dolor físico que pudieran estar afectando el trabajo habitual y actividades de la vida diaria de las educadoras.

Respecto a la salud general, relacionada con la percepción que se tiene sobre el estado de salud personal y en relación a otros, se aprecia como regular (60 puntos), el 62,2\% ( $n=97)$ tiene regular y el 34\% ( $n=53$ ) bueno, lo que probablemente indica que la valoración personal del estado de salud (percepción de la situación actual y futura) no es tan adecuada.

Por otra parte, la vitalidad, aspecto referido al ánimo o energía con el que se enfrenta el día a día, es percibida como regular (57 puntos), no tiene casos en muy malo ni óptimo, un 68,6\% (n = 107) está regular y un 29,5\% ( $\mathrm{n}=46)$ está en bueno, lo que podría involucrar la falta de energía, cansancio y desánimo en las actividades cotidianas.

La función social, que implica la percepción que se tiene respecto a cómo los problemas físicos o emocionales interfieren en las actividades sociales, también es percibidas como regular (58 puntos), no presenta casos muy malos ni óptimos, el 73,1 \% (n = 114) está regular, el $17,3 \%(\mathrm{n}=27)$ está bueno y el 9,6\% $(\mathrm{n}=15)$ está malo, situación que posiblemente implica que los problemas físicos y/o emocionales derivados de la falta de salud estarian interfiriendo en la vida social habitual de las profesionales.

El Rol emocional, referido al grado de interferencia de los problemas emocionales en la vida diaria y profesional, es percibido como óptimo (88 puntos). No hay casos malos un muy malos. El $75,6 \%(n=118)$ presenta óptimo y el $11,5 \%(n=18)$ es regular, situación que podría implicar que las educadoras logran diferenciar los problemas del ámbito personal con los aspectos laborales. Finalmente, la Salud mental, relacionada con la valoración general del estado de salud mental, considerando aspectos como la depresión, la ansiedad, el autocontrol y el bienestar general, se aprecia como buena (69 puntos). No hay casos muy malos, el 85,3\% ( $\mathrm{n}=$ 133) es bueno y el 10,3\% $(n=16)$ es regular. Situación que reflejaría que en general, las educadoras manifiestan un buen estado de salud mental que no interfiere en su desempeño profesional.

\section{Análisis correlacional}

Quienes presentan óptima función física se caracterizan mayoritariamente por: tener entre 23 y 35 años $(46 \%, n=63)$, llevar entre 1 y 10 años laborales $(48,9 \%, \mathrm{n}=67)$, tener entre 21 a 40 niños a cargo $(62 \%, n=85)$, presentar entre 0 a 5 dias de ausentismo $(50,4 \%, n=69)$, se desempeñan en el sistema particular subvencionado $(46,7 \%, \mathrm{n}=64)$, y desempeñarse en los niveles de transición $(69,3 \%, \mathrm{n}=95)$.

Existiendo una relación altamente significativa entre la función física con los dias de ausentismo laboral (chi-cuadrado de Pearson $17,46 ; p=0.008)$ y con la dependencia laboral ((chi-cuadrado de Pearson 15,$44 ; \mathrm{p}=0.004)$. Similar, pero con una relación signifi- 
cativa, con la cantidad de niños a cargo (chi-cuadrado de Pearson 10,53; $\mathrm{p}=0.032$ ) y con los años laborales (chi-cuadrado de Pearson 11,68; $\mathrm{p}=0.020$ ).

En el rol físico, quienes lo presentan como óptimo se caracterizan mayoritariamente por: tener entre 23 y 35 años $(45 \%, n=59)$, llevar entre 1 y 10 años laborales $(48,1 \%, \mathrm{n}=63)$, tener entre $21 \mathrm{a}$ 40 niños a cargo $(60,3 \%, \mathrm{n}=79)$, presentar entre 0 a 5 días de ausentismo (51,1\%, $n=67)$, desempeñarse en el sistema particular subvencionado $(46,6 \%, \mathrm{n}=61)$ y se desempeñan en los niveles de transición $(72,5 \%, n=95)$. No existiendo relación significativa entre las variables sociodemográficas y el Rol físico.

Quienes se clasifican como muy malo en dolor corporal se caracterizan mayoritariamente por: tener entre 23 y 35 años (51,2\%, n = 22), llevar entre 1 y 10 años laborales $(55,8 \%, n=24)$, presentar de 0 a 5 dias de ausentismo $(69 ; 8 \%, \mathrm{n}=30)$, desempeñarse en los niveles de transición $(69,4 \%, \mathrm{n}=29)$ y tener entre 21 a 40 niños a cargo $(68,1 \%, n=32)$. Existiendo una relación altamente significativa entre el dolor corporal con los dias de ausentismo laboral (chi-cuadrado de Pearson 26,57; $\mathrm{p}=0.009$ ).

En relación a la salud general, quienes la refirieron como regular se caracterizan mayoritariamente por: tener entre 23 y 35 años (43,3\%, $n=42)$, llevar entre 1 y 10 años laborales $(47,4 \%, n=46)$, entre 21 a 40 niños a cargo $(60,8 \% \mathrm{n}=59)$, presentar de 0 a 5 días de ausentismo $(48,5 \%, n=47)$, desempeñarse en el sistema particular subvencionado $(49,5 \%, \mathrm{n}=4$ 8) y trabajar en los niveles de transición (71,1\%, $n=69$ ). No existiendo relación significativa entre las variables sociodemográficas y la salud general.

La vitalidad referida como regular, se caracterizó en los participantes mayoritariamente por: tener entre 23 y 35 años $(39,3 \%, n=42)$, llevar entre 1 y 10 años laborales (43,9\%, $n=47)$, tener entre 21 a 40 niños a su cargo $(60,7 \%, \mathrm{n}=65)$, presentar de 0 a 5 días de ausentismo $(45,8 \%, n=49)$, desempeñarse en el sistema particular subvencionado $(42,1 \%, \mathrm{n}=45)$ y trabajar en los niveles de transición $(74,8 \%, \mathrm{n}=80)$. La vitalidad se relacionó significativamente con la cantidad de niños a cargo (chi-cuadrado de Pearson 9,98; $p=0.041$ ). Quienes se clasifican como regular en función social se caracterizaron mayoritariamente por: tener entre 23 y 35 años $(41,2 \%, n=$ 47), llevar entre 1 y 10 años laborales (43,9\%, n = 50), tener entre 21 a 40 niños a cargo $(59,6 \%, n=68)$, presentar entre 0 a 5 días de ausentismo (50\%, $n=57)$, desempeñarse en el sistema particular subvencionado $(47,4 \%, \mathrm{n}=54)$ y trabajar en los niveles de transición $(78,1 \%, n=89)$. Existiendo una relación altamente significativa entre la función social con el nivel de desempeño (chi-cuadrado de Pearson $17,27 ; \mathrm{p}=0.002)$.

En el rol emocional, quienes se clasifican como óptimo se caracterizan mayoritariamente por: tener entre 23 y 35 años $(40,7 \%, n=48)$, llevar entre 1 y 10 años laborales $(45,8 \%, n=54)$, tener entre 21 a 40 niños a cargo $(61 \%, \mathrm{n}=72)$, presentar entre 0 a 5 días de ausentismo (52,5\%, n = 62), desempeñarse en el sistema particular subvencionado $(44,9 \%, n=53)$ y trabajar en los niveles de transición $(74,6 \%$, $\mathrm{n}=88$ ). El rol emocional se relaciona significativamente con los dias de ausentismo (chi-cuadrado de Pearson 14,73; $p=0.022$

Por último, quienes se clasifican como buena en salud mental se caracterizan mayoritariamente por: tener entre 23 y 35 años (40,6\%, $n=54)$, llevar entre 1 y 10 años laborales $(43,6 \%, n=58)$, tener entre 21 a 40 niños a su cargo $(58,6 \%, n=78)$, presentar de 0 a 5 días de ausentismo (48,9\%, $n=65)$, desempeñarse en el sistema particular subvencionado $(44,4 \%, n=59)$ y trabajar en los niveles de transición $(72,2 \%, n=56)$. No existiendo relación significativa entre las variables sociodemográficas y la salud mental.

\section{DISCUSIÓN}

Muchos estudios dan a conocer la importancia de la atención y educación de la niñez en la primera infancia, para generar cambios significativos en el desarrollo de las personas. Es por ello que el interés político en la educación parvularia justifican aunar los esfuerzos e inversiones estatales, potenciando la inclusión de los menores de 0 a 6 años en el sistema escolar, fundamentándose que en el primer año de vida suceden la mayoría de los cambios a nivel cerebral, los niños y niñas necesitan de experiencias cognitivas y afectivas positivas para garantizar un desarrollo adulto adecuado, y la importancia de promover espacios educativos de alta calidad para asegurar un desarrollo integral. ${ }^{28-32}$

Por lo tanto, la incidencia que tienen los docentes o educadores en los primeros años es determinante en la maduración de las estructuras biológicas y el desarrollo humano. En sintonía con ello, en 1990 Chile se compromete a garantizar un conjunto de condiciones para el desarrollo de la infancia, en el marco de la convención de Derechos de Niño, y por medio de políticas públicas, dispositivos legales e instituciones que lo hagan exigible. ${ }^{33}$

En base a ello, en los últimos 20 años se han generado políticas gubernamentales orientadas a fortalecer el sistema de educación parvularia, con enfoque en la cobertura y calidad de la misma, abocando las acciones hacia un sistema de acreditación educativa y evaluación de los aprendizajes, considerando que la calidad del servicio educativo se asocia a beneficios en el ámbito cognitivo y socioemocional de los niñas y niños, y actualmente precisa apenas un $26 \%$ de cobertura. ${ }^{34-36}$

Los educadores de párvulos son agentes especializados en la educación de los niños de entre 0 a 6 años, por lo tanto, tienen un rol fundamental en la conformación de un ambiente educativo propicio, incidiendo en la calidad de educación que proporcionan, entre otros aspectos, los estudios, las capacitaciones y las condiciones de trabajo. Actualmente ésta calidad se ha visto sumamente cuestionada, pues un estudio en 120 centros de educación parvularia en Chile puso en manifiesto que, independiente de su condición de institución pública o privada y de las diferencias socioeconómicas existentes entre estos, todos presentaron un promedio de rango mínimo en calidad, con niveles de apoyo pedagógico bajos. ${ }^{37,38}$

De este modo, se hace imprescindible profundizar sobre las características propias de las educadoras y cuáles de estos factores personales y de calidad de vida están relacionados con la salud, y analizar con ello cómo mejorar la calidad de educación y herramientas cognitivas y emocionales significativas para los niños y niñas del país. En este esfuerzo, el estudio arroja condiciones similares a otras indagaciones sociodemográficas de las educadoras; en general, estamos refiriéndonos a una profesión marcada por el género femenino, en su mayoría jóvenes menores a 40 años, con baja participación en los niveles de sala cuna y niveles medios en concordancia con la baja cobertura aún del sistema en estos niños y niñas.

La experiencia laboral en varios estudios coincide con una cantidad no menor de 12 años, con prácticamente la totalidad desempeñándose en el aula, con alrededor de 26 párvulos a su cargo, y con una satisfacción mayor relacionada a las condiciones generales del establecimiento y el clima laboral, medianamente adecuada e inadecuada con la remuneración, incentivos y beneficios económicos, recordando que este tipo de profesional docente es el peor pagado en nuestro país y con altos niveles de estrés. ${ }^{36,39,40,17-19}$

Una de las pruebas que denotan la falta de condiciones laborales decentes, en concordancia con su carácter profesional y exigencias 
de calidad, es la presencia de ausentismo laboral por motivos de enfermedad, que no siempre sus diagnósticos están directamente relacionadas a lo ocupacional, pero sí precisan causantes relacionados a las actividades diarias involucradas en el trabajo. ${ }^{41} \mathrm{La}$ calidad de vida relacionada con la salud mostrada en el estudio no precisa datos alarmantes, pero sí de importancia para garantizar un desempeño óptimo en el aula con, por ejemplo, referir una salud general, vitalidad y funciones sociales regulares. Condiciones que son apoyadas por otros estudios que describen en las educadoras de párvulo agotamiento emocional, menor satisfacción laboral e inclusive burnout. ${ }^{36,39,41}$
Muchos de los hallazgos revelados en el estudio asocian la calidad de vida, relacionada con la salud, con la cantidad de niños a cargo, el nivel en que se desempeñan y su dependencia laboral, además de los días de ausentismo en el trabajo. Condiciones que podrian ser determinantes en la orientación de las prácticas educativas en el aula y ponen en manifiesto que las nuevas disposiciones y politicas gubernamentales han dejado un vacío en asegurar condiciones que propicien una buena calidad de vida docente, que proteja su tan determinante e influyente salud física, mental, social y emocional en los niños y niñas a los cuales se les exige educar con calidad.

\section{REFERENCIAS}

1. Patián J. Construcción y propiedades psicométricas de la Escala de Calidad de Vida en el Trabajo. Cienc Trab. 2016; 18(56):94-105.

2. Iglesias-Fernández $C$, Llorente-Heras $R$, Dueñas-Fernández D. Calidad del empleo y satisfacción laboral en las regiones españolas. Un estudio con especial referencia a la Comunidad de Madrid. Invest Region. 2011;(19):25-49.

3. Martínez-Gamarra M, Ros Mar R. De la calidad de vida laboral a los riesgos psicosociales: evaluación de la calidad de vida laboral. Acc Invest Soc. 2010;(28):5-55.

4. WHOQOL Group. Study protocol for the World Health Organization Project to developed a quality of life assessment instrument (WHOQOL). Qual Life Res. 1993; 2(2):153-159.

5. Vélez C, Escobar M, Pico M. Calidad de vida y trabajo informal en personas mayores en una ciudad intermedia colombiana, 2012-2013. Rev Bras Geriatr Gerontol. 2016; 19(3):415-427.

6 Schawartzmann L. Calidad de vida relacionada con la salud: Aspectos conceptuales. Cienc enferm. 2003; 9(2):9-21.

7. Dulcey $\neg$ Ruiz $E$, Quintero G. Calidad de vida y envejecimiento. En: Rocabruno JC. Tratado de Gerontología y Geriatría Clínica. Ciudad de La Habana: Científico-Técnica; 1999.
8. Quintero G. Calidad de vida y envejecimiento. En: Prieto O, Vega E. Temas de Gerontología. Ciudad de La Habana: Científico-Técnica; 1996.

9. Mantilla G, Márquez A. Envejecimiento: Experiencias y perspectivas. Bogotá: AIG de Colombia; 1995.

10. Castel R. El ascenso de las incertidumbres: trabajo, protecciones y estatuto del individuo. México: Fondo de Cultura Económica; 2010.

11. Garduño L, Salinas B, Rojas M. Calidad de vida y bienestar subjetivo en México: Plaza y Valdés; 2005.

12. Hernández-Vicente $I$, Lumbreras-Guzmán $M$, Méndez-Hernández $P$, RojasLima $E_{1}$ Cervantes-Rodríguez $M$, Juárez-Flores $C$. Validación de una escala para medir calidad de vida laboral en hospitales públicos de Tlaxcala. Salud Públ Méx. 2017; 59(2):183-192.

13. Blanch-Josep $M$, Sahagún $M$, Cervantes G. Estructura factorial del Cuestionario de Condiciones de Trabajo. Rev Psicol Trabajo Organ. 2010; 26(3):175-189.

14. Mesa A, Gómez A. Salud mental, función docente y mentalización en la Educación Preescolar. Psicol Escol Educ. 2015; 19 (1):117-125.

15. Cornejo R. Salud laboral docente y condiciones laborales: Entre el sufrimiento individual y los sentidos colectivos. Rev Docencia (Santiago de Chile) 


\section{REFERENCIAS}

[en línea]. 2008 [consultada may 2017]; (35):77-85. Disponible en: http:// www.revistadocencia.cl/new/wp-content/pdf/20100730180726.pdf.

16. Claro S, Bedregal P. Aproximación al estado de salud mental del profesorado en 12 escuelas de Puente Alto. Rev méd Chile [en línea]. 2003 [consultado may 2017]; 131(2):159-167. Disponible en: https://scielo.conicyt.cl/scielo. php? script=sci_arttext\&pid=S0034-98872003000200005

17. Salanova M. Psicología de la salud ocupacional. Madrid: Sintesis; 2009.

18. Eyzaguirre S. ¿Son atractivos los salarios de la nueva carrera docente? Puntos de Referencia-CEP [en línea]. 2015 [consultado may 2017]; (401):00-08 Disponible en: https://www.cepchile.cl/cep/site/artic/20160304/asocfile/201 60304101259/pder401_SEyzaguirre.pdf.

19. Hierbert BA, Farber I. Teacher stress: literature survey with a few surprises. Can J Educ. 1984; 9(1):14-27.

20. Junta Nacional de Jardines Infantiles-JUNJI. Informe Metas de Cobertura. Programa Meta Presidencial [en línea]. Santiago de Chile: Senado de la República; 2017 [consultado may 2017]. Disponible en: http://www.senado. cl/site/presupuesto/2017/cumplimiento/Glosas\%202017/cuarta_subcomision/09\%20Educaci\%C3\%B3n/ORD.\%20015-0759\%20JUNJI\%202017.pdf

21. Chile. Ministerio de Educación. Crea la Subsecretaria de Educación Parvularia, la Intendencia de Educación Parvularia y modifica diversos cuerpos legales. Ley 20.835 (28 abr 2015). Santiago de Chile: MINEDUC; 2015.

22. Olivares-Tirado P. Estado de Salud de Beneficiarios del Sistema de Salud de Chile: 2004 -2005 (documento de trabajo) [en línea]. Santiago: Gobierno de Chile-Superintendencia de ISAPRES; 2006 [consultado may 2017]. 40 p. Disponible en: http://www.supersalud.gob.cl/documentacion/569/articles-10 62_recurso_1.pdf.

23. Chile. Ministerio de Salud. División de políticas saludables y promoción. Protocolo de vigilancia para trabajadores expuestos a factores de riesgo de trastornos musculoesqueléticos de extremidades superiores relacionados con el trabajo [en línea]. Santiago: MINSAL; 2012 [consultado may 2017]. Disponible en: http://web. minsal.cl/portal/url/item/dbd6275dd3c8a29de 040010164011 886.pdf.

24. McHorney C, Ware J, Raczek A. The MOS 36-Item Short-Form Health Survey (SF-36): II. Psychometric and Clinical Tests of Validity in Measuring Physical and Mental Health. Med Care. 1993; 31(3):247-63.

25. Mosqueda A, Puebla P, Barriga O. Evaluación psicométrica de la escala SF-36 en personas con insuficiencia renal en tratamiento con hemodiálisis. Rev iberoam educ invest enfer-ALADEFE. 2013; 3(4):29-36.

26. Dois $A$, Contreras A. Validación de una escala de calidad de vida en un grupo de personas con esquizofrenia de la región metropolitana, Chile. Cienc enferm. 2007; 13(1):35-44.

27. Rodríguez-Romero B, Pita S, Pertega S. Calidad de vida relacionada con la salud en trabajadoras del sector pesquero usando el cuestionario SF-36. Gac Sanit. 2013; 27(5):418-424.

28. Peralta MV. Nacidos para ser y aprender. Buenos Aires: Infanto-juvenil; 2005.

29. Zapata B, Ceballos L. Opinión sobre el rol y perfil del educador para la primera infancia. Rev Latinoam Cienc Soc Niñez Juv. 2010; 8(2):1069-1082.

30. Elvir AP, Asensio C. La atención y educación de la primera infancia en Centroamérica: Desafíos y perspectivas [en línea]. Paris: UNESCO; 2006 [consultado may 2017]. Disponible en: http://unesdoc.unesco.org/ images/0014/ 001474/147488s.pdf.

31. Eming $M$, Fujimoto $G$. Desarrollo infantil temprano: Lecciones de los programas no formales. Acción Pedagóg. 2004; 13(2):186-198.

32. Myers R. Atención y desarrollo de la primera infancia en Latinoamérica y el Caribe: Una revisión de los diez últimos años y una mirada hacia el futuro. Rev Iberoam Educ. 1999; 22:17-39.

33. Chile. Ministerio de Relaciones Exteriores. Promulga Convención de los Derechos del Niño. Decreto 830 (27 sept 1990.) Santiago de Chile: Ministerio de Relaciones Exteriores; 1990.

34. Centro de Estudios MINEDUC. Impacto de asistir a Educación Parvularia. Evidencias para políticas públicas en educación. Compilación Serie Evidencias 2012-2013. Santiago de Chile: MINEDUC; 2013.

35. Chile. Ministerio de Educación. Potenciar el talento de nuestros niños. Educación Preescolar. Estrategias Bicentenario. Santiago de Chile: MINEDUC; 2005.

36. Bravo A. Formación especializada del educador de párvulos y calidad educativa del ambiente en salas del nivel sala cuna. Rev Invest (Caracas). 2015; 86 (39):63-82.

37. Pizarro P, Espinoza V. ¿Calidad en la formación inicial docente? Análisis de los nuevos estándares de la educación de párvulos en Chile. Perspec EducFormac Prof. 2016; 55(1):152-167.

38. Treviño, E., Toledo, G. \& Gempp, R. Calidad de la educación parvularia: las prácticas de clase y el camino a la mejora. Pensamiento Educativo. Rev Invest Educ Latinoam. 2013; 50(1):40-62.

39. Bravo A, Morales H. Caracterización de educadoras de párvulos que se desempeñan en el nivel sala cuna en una muestra de jardines infantiles de Concepción, Chile. Rev Actual Investig Educ. 2012; 12(2):1-25.

40. Ortiz M, Castelvi M, Espinoza L, Guerrero R, Lienqueo P, Parra R, Villagra E. Tipos de personalidad y sindrome de burnout en educadoras de párvulos en Chile. Univ Psychol. 2012; 11(1):229-239.

41. Guerrero E. Análisis pormenorizado de los grados de burnout y técnicas de afrontamiento del estrés docente en profesorado universitario. An Psicol. $2003 ; 19: 145-158$. 